\title{
Low cycle fatigue numerical estimation of a high pressure turbine disc for the AL-31F jet engine
}

\author{
Miroslav Spodniak ${ }^{1, *}$, Marek Klimko ${ }^{2}$, Marián Hocko ${ }^{1}$, and Pavel Žitek ${ }^{2}$ \\ ${ }^{1}$ Technical University of Kosice, Department of Aviation Engineering, 7 Rampová, 04221 Košice, Slovakia \\ ${ }^{2}$ University of West Bohemia, Department of Power System Engineering, 22 Univerzitní, 30614 Pilsen, Czech Republic
}

\begin{abstract}
This article deals with the description of an approximate numerical estimation approach of a low cycle fatigue of a high pressure turbine disc for the AL-31F turbofan jet engine. The numerical estimation is based on the finite element method carried out in the SolidWorks software. The low cycle fatigue assessment of a high pressure turbine disc was carried out on the basis of dimensional, shape and material disc characteristics, which are available for the particular high pressure engine turbine. The method described here enables relatively fast setting of economically feasible low cycle fatigue of the assessed high pressure turbine disc using a commercially available software. The numerical estimation of accuracy of a low cycle fatigue depends on the accuracy of required input data for the particular investigated object.
\end{abstract}

\section{Introduction}

A low cycle fatigue (LCF) is a very common task not only in engineering industry but in aviation industry as well. The first LCF's occurrence in aviation industry along with its association with new technologies and new materials has demanded the requirement for a special attention dedicated to this theme. As it is well known, the LCF of jet engines is linked with more factors and effects.

A jet engine is a heat machine in which thermal energy is delivered from the combustion of fuel with air and consequently converted to useful work. [1] A jet engine and its constructional parts are subject to very high loads and moments, which depend on the engine regime. Considering the angle of changing load in jet engine parts in dependence on time, it is possible to distinguish two kinds of loads; quasi static and dynamic load. In calculation, it is necessary to examine each part individually because of a variety of the load. Jet engine parts and construction assemblies are subject to load and aerodynamic forces, which are due to streaming of air and hot burning gases through the engine sections. The actual pattern of aerodynamic force depends on the difference of pressures and a variation of momentum. This force has two compounds, axial and tangential. Other load is caused by centrifugal load and momentum, which arise by the movement of mass and fly maneuvers. [2] On rotating parts of jet engine, centrifugal forces emerge, which have influence on the LCF. Centrifugal forces are subject to the rotor and its parts, such as a shaft, a disc, a compressor and turbine blades etc. Spinning of these parts sets off centrifugal forces, which have to be included in numerical counts. Another meaningful factor for counting a life estimation is temperature. A mixture of fuel and compressed air is burned in a combustion chamber under very high temperature. From the combustion chamber, hot gases flow across high pressure and low pressure turbine to exhaust a nozzle. Besides transition, these hot gases create thermal stress, which has the effect on cooling the pressure turbine disc and cooling the turbine rotor blades. [3] The condition of jet engine parts influence several factors, either directly or indirectly. It also includes the atmospheric conditions, in which a jet engine is operated, then the speed of flight, flight level etc.

Jet engine parts load, as it is known, is related to high temperatures. The fatigue of a pressure turbine disc is caused by cyclic changes of load, which are induced by the temperature gradient, centrifugal forces of spinning parts of the disc and rotor blades as well. Cyclic temperature relief has a significant impact during transitional regimes of a jet engine. These varieties of the temperature cause thermal fatigue of material, the beginning of a disc cracks and their gradual increasing.

The most unfavourable effect of cyclic fatigue results in the shutdown of a jet engine in high altitudes with a subsequent autorotation. This process is associated with severe cooling down of hot jet engine parts. As a result, this process creates very a high strain inside and outside the material. Standardly, the highest thermal gradients are reached in time from five to ten minutes, from the beginning of a transitional process. The cycle fatigue is linked and accompanied also with jags, holes, disc rims, in which stress is concentrated. 


\section{Constructional characteristic of the pressure turbine disc AL-31F jet engine}

A pressure turbine of a bypass turbofan jet engines like $\mathrm{AL}-31 \mathrm{~F}$, that is used in Su-27 aircraft, is axial, two shafts. The pressure turbine consists of a one stage high pressure turbine and a one stage low pressure turbine. Both of them have rotor blades and stator vanes mounted, which are cooled by air. On lower regimes it is for the purpose to increase the engine thrust used partly for disconnection of cooling both of pressure turbines. [4]

A high pressure turbine consists of a disc, rotor blades, gudgeon and a high pressure turbine shaft.

A high pressure turbine disc is made by ironwork with consequent chip machining. The disc inner rim rabbets are made in order to ensure attaching rotor blades. There are 90 rotor blades with rabbets to locate elastic blade locks given to ensure axial movement and slant holes for cooling the air input. On the right side of the disc, there is located a labyrinth seal rim and a rim, which is used to remove disc. In the disc flange section there are roll holes drilled to fit screws that are connecting the shaft, the disc and the high pressure turbine rotor pivot. The high pressure turbine AL-31F prime parameters:

- The overall ratio of expansion: $\pi_{T c}=2.9$.

- Top efficiency: $\eta_{T c}=0.87$.

- Circumferential velocity: $u=510 \mathrm{~m} \cdot \mathrm{s}^{-1}$.

- RPM: $n=13300$ min. $^{-1}$.

- The temperature of the inlet gas: $T_{3 c}=1665 \mathrm{~K}$.

- Weight: $G=70 \mathrm{~kg}$.

- Speed ratio: $u / c_{1}=0.46$.

- High pressure turbine gas flow rate: $Q_{p l . V T T}=70 \mathrm{~kg} . \mathrm{s}^{-1}$.

- Low pressure turbine gas flow rate: $Q_{\text {pl.NTT }}=72 \mathrm{~kg} . \mathrm{s}^{-1}$.

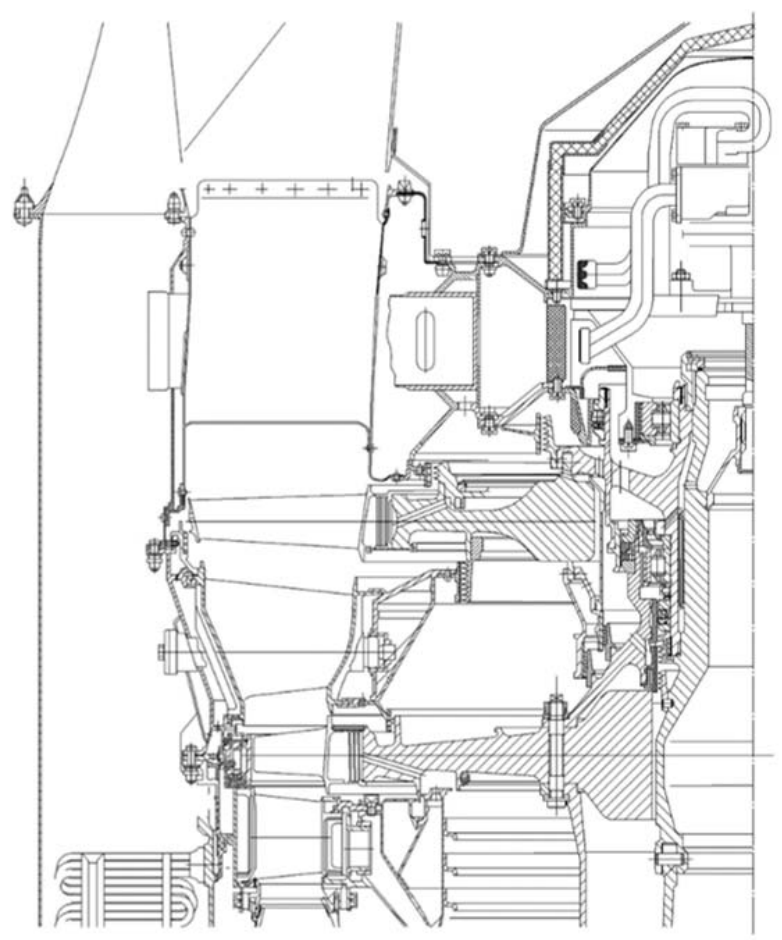

Fig. 1. Two stage pressure turbine configuration

\section{Geometry and finite element model of the AL-31F high pressure turbine disc}

The first step in the life evaluation of the jet engine AL$31 \mathrm{~F}$ high pressure turbine disc was a creation of a $3 \mathrm{D}$ model in the CAD program. Modeling was carried out by Solid modeling in 3D program SolidWorks. The program SolidWorks allows Solid modeling and to execute needed analysis to use for a life fatigue counting. A life cycle fatigue analysis of AL-31F jet engine high pressure turbine disc was carried out by the finite element method in SolidWorks. The information from the available resources and technical documentation were used to create an identical 3D high pressure turbine model of the AL-31F jet engine. In order to simplify the calculation, the segment from 3D modeling was formed. The segment is shown on the Fig. 2; the meshing of the segment was made in SolidWorks program, too. In order to be able to perform static analyses in SolidWorks simulation, it is necessary to apply boundary conditions (Fig. 3). [5]


Fig. 2. Geometry and finite element model

The life fatigue calculation of the jet engine AL-31F high pressure turbine has been simplified in terms of a commercial engine mission. Two fundamental regimes of a jet engine have been used, idle run and maximal regime. As well as engine regimes, material properties of 
the disc are necessary. Other simplification lay in material properties dependent on temperature.

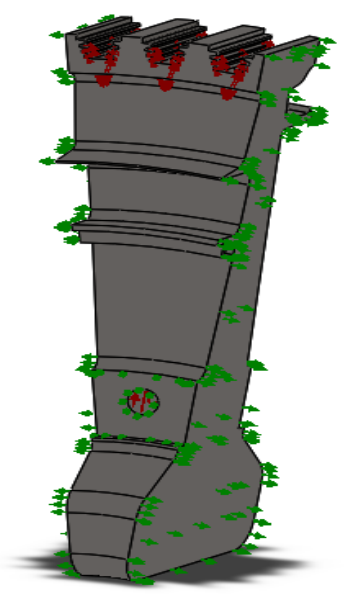

Fig. 3. Boundary conditions of the disc element

The static analyses have been carried out in the finite element method; these analyses dictate the stress of the disc that is operated on the idle run and maximal regime. Before we execute any analyses, it is necessary to find out the forces system of the disc, as described in the first chapter. The forces system of the jet engine AL-31F high pressure disc is shown in the Fig. 4. For the purpose of this numerical estimation, there were two thermal analyses proceeded - "thermal 1" and "thermal 2". The thermal analysis marked as "thermal 1" represents the heat load of the disc running on the idle run. The thermal analysis "thermal 2" defines the heat load on the maximal regime. In Fig. 4, we can see forces system of the AL-31F disc, where its resulting load is produced by the resulting centrifugal load caused by blades $F_{o}$, centrifugal load of the disc, centrifugal load of screws $F$, then axial forces on screws $F_{A}, F_{t}$ friction force, $M_{t}$ torque flange, $P_{s}$ overlap pressure of screws. [3], [6]
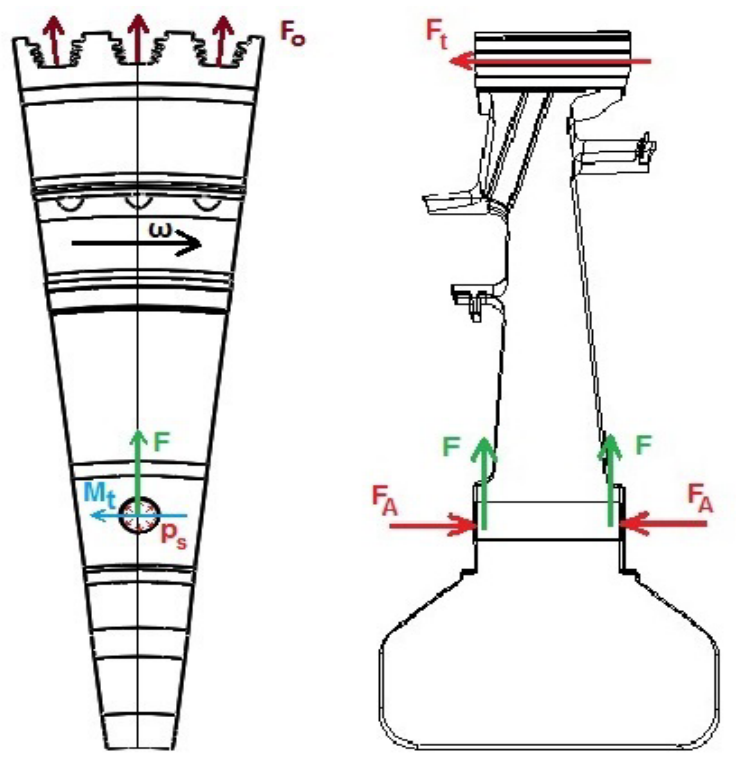

Fatigue strength properties are necessary to evaluate the total life, according to the mathematical formula:

$$
\frac{\Delta \varepsilon}{2}=\underbrace{\frac{\sigma^{\prime}}{E}\left(2 N_{f}\right)^{b}}_{\text {ELASTIC }}+\underbrace{\varepsilon_{f}^{\prime}\left(2 N_{f}\right)^{c}}_{\text {PLASTIC }}
$$

The fatigue failure under LCF is based on a characteristic plastic deformation:

$$
\frac{\Delta \varepsilon}{2}=\varepsilon_{f}^{\prime}\left(2 N_{f}\right)^{c}
$$

where:

$-\Delta \varepsilon$ : local strain range

- $\sigma^{\prime}$ : cyclic stress amplitude

- E: Young's modulus

- $c$ : fatigue ductility exponent

- $N_{f}$ : number of cycle to failure

- $\varepsilon$ : fatigue ductility coefficient [5]

\section{Thermal analysis of the AL-31F high pressure turbine disc}

As discussed in the previous section, temperature has a major effect on LCF. The disc is subject to high thermal gradients, which are given by the difference of high temperature. Another influence brings about the fact, that the disc is cooled by air from the compressor. The temperature of the disc $\mathrm{AL}-31 \mathrm{~F}$ jet engine achieves values within $370^{\circ} \mathrm{C}$ till $590^{\circ} \mathrm{C}$ on the maximal regime. The biggest thermal difference occurs between the outer rim, respectively in a part of the blade mounting and the inner rim. Shutting down the jet engine in high altitude has a particularly dangerous impact, when the engine parts with high temperature are sharply cooled, and vice versa, after repeated shutting down, they are swiftly heated, which results in the induction of an extremely high load. In order the disc to be totally overheated for the working temperature, the required period is 5 to 10 minutes, after finishing the transition process.

The temperature distribution at the radius of the disc and its temporal changes is dependent on constructional parameters rather than on the method of cooling, on the perfection of the jet engine regulatory system and flight cycles of the jet engine. During the starting process, the thermal gradients grow at the radius of the disc and are associated with growing of compressive stress and thermal stress in the disc rim, which is heated faster. In the central part of the disc, which has a higher thermal inertia, tensile stress arises on a start-up.

The thermal analyses for the purposes of this article have been carried out twice for two different regimes; first it is the idle run and, second, the maximal regime. In this task, the numerical estimation has been simplified with the temperature of the sectors at the disc surface. The result of the thermal analysis is shown in the picture 5 , in terms of the temperature dependence and the diameter of the disc.

Fig. 4. Forces system AL-31F disc element 

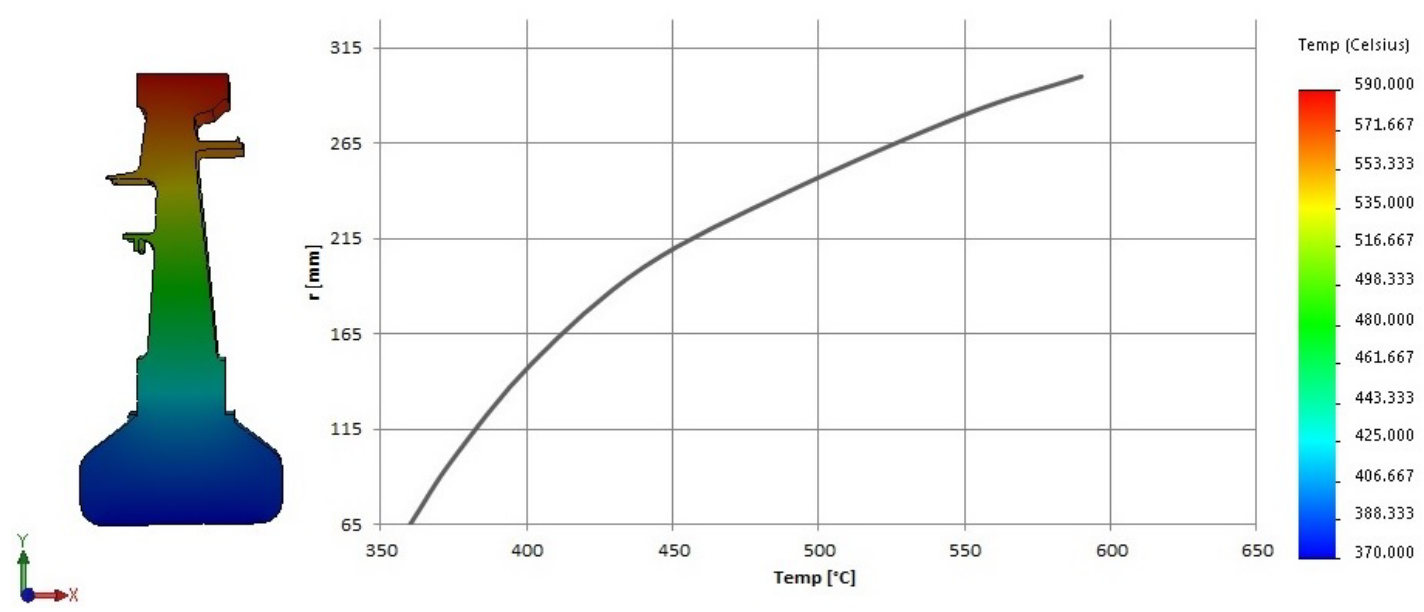

Fig. 5. Temperature profile

\section{Finite element method analyses}

The finite element method analysis (FEA) for life fatigue determination has been carried out. In this analysis, information acquired in thermal and structural studies has been used, as discussed in the previous sections. These studies are in accordance with the idle run and the maximum regime. Thermal load combined with a centrifugal load of a disc, the centrifugal load of blades, screws and other loads were counted in two strength analyses the for idle run and maximal regime as well as for thermal analyses.

The most critical values of the reduced stress have been occurring in both static analyses, where the most critical section was concentrated on the edge of the disc flange in the inner rim and in holes for mounting screws. The outcome of this study is represented by Fig. 6 .
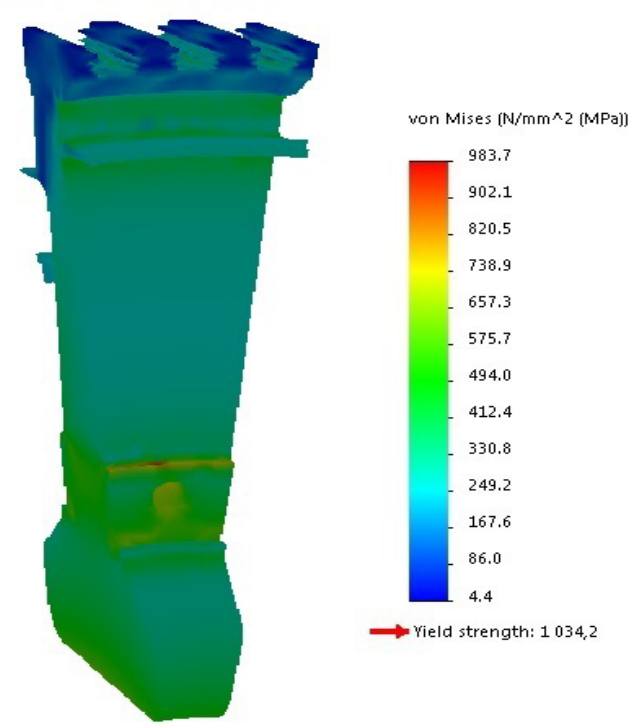

Fig. 6. Stress of von Mises

\section{Fatigue life assessment of the AL-31F jet engine high pressure turbine disc}

The strain and stress obtained from static analyses are used for LCF life analyses. In order to carry out the life analysis, there is another information required. Apart from linear static analyses, there is material information, which is necessary for life analyses, such as Yield Strength, Tensile Strength, Young Modulus, Poisson ratio and SN curve. For the purposes of this study, the data was generated by 3D CAD software. The cyclic load is based on a periodic load between the two static analyses, the idle run and the maximal regime.



Fig. 7. Total life

The linear static analyses correspond with started regimes in CAD software, which provides information about the most stressed part of the disc, thus the highest concentration of the reduced strain in the disc. This information is necessary for a life estimation along with other material information, as discussed previously.

The result of the fatigue analysis has indicated a number of cycles, which overrun, and would 
theoretically mean the beginning of the fatigue crack propagation. In the section view, there are shown the most critical parts of the element disk, which acquire the minimum number of cycles. In Figure 7, there is clearly shown the most critical part of AL $31 \mathrm{f}$ engine disc. Thus the hole for a screw is the most critical part, which reaches minimum Total Life to crash. The most critical load is concentrated in the section of the disc flange.

\section{Conclusion}

The exact determination of the LCF jet engine high pressure disc, in particular the type of the jet engine, largely affects the economic efficiency of the jet engine.

The accuracy of results obtained from the static analyses and from the life fatigue study of the AL-31F jet engine high pressure disc is slightly influenced in terms of some simplifications, which have been discussed in the previous sections. For an accurate evaluation, essential exact data are required, most of which it is possible to acquire just from a manufacturer. As for temperature data, this is the most important fact for the LCF disc evaluation. To by exact in the life estimation, it is necessary to have data about temperature of the sectors at the disc surface. Despite these facts, this article presents a procedure of LCF estimation for a concrete jet engine and states critical sections of a high pressure disc.

LCF determination is a demanding and lengthy process, numerical counts not always correspond with real situations. The accuracy of the LCF estimation is possible to verify by practical tests, in which there are simulated real conditions of high pressure turbine operating. Such tests are compared with the numerical method of LCF determination more demanding in terms of time as well as economic and ecological viewpoint, but despite this fact in practice, these tests have to be executed.

This work has been supported by the project SGS-2016-045 (Increasing the efficiency, reliability and service life of power system machines and devices 4) and the Ministry of Education, Youth and Sport Czech Republic Project LQ1603 (Research for SUSEN). This work has been realized within the SUSEN Project (established in the framework of the European Regional Development Fund (ERDF) in project CZ.1.05/2.1.00/03.0108).

\section{References}

1. F.C. Rogers, Y.R. Mayehew, Gas turbine theory $4^{\text {th }}$ edtion (London, 1996), ISBN 0-582-23632-0

2. J. Kamenický, Z. Linhart, Konstrukce leteckych motoru II. (Brno, 1989)

3. J.L. Kerrebrock, Aircraft engines and gas turbines II (Massachusetts Institute of Techonology, 1992)

4. S.V. Falaleev, Construction AL-31F turbofans (Samara)

5. M. Vable, Mechanics of materials II (Michigan Technological University, 2012)

6. P.P. Walsh, P. Fletcher, Gas turbine performance (2004), ISBN 0-632-06434-X
7. K. Semrád, Pevnostná analýza disku plynovej turbíny motora DV-2 (Košice, 2001) 